\title{
Fusion of Infrared and Visible Images for Surveillance Applications using Ann
}

\author{
Bhavatharane K, Minal Moharir
}

\begin{abstract}
Edgepreserving filters were used to Multi-Scale Decomposition (MSD) for fusion of visible and infrared images.Traditional edgepreserving MSDs may be unable to achieve satisfactory structural separation from details, resulting in fusion performance degradation. The objective of this work is to propose a MSDIteration with ANN fusion technique for infrared and visual image which improves the fusion execution. Initially the original image is decomposed by "Gaussian smoothness and joint bilateral filter". Edge retention and scaling perception attributes are introduced to satisfactorily separate image details from source images. Decomposition includes preserving the attributes of edge and zoom perception, so that the detail information is completely separated from the image details and the improvement of fusion performance is maintained. The rule aims to merge these decomposed layers. A saliency map is constructed by Laplacian and Gaussian low-pass filtersto find the initial weight mapand further a guided filter is used to determine the final weight map. The enhanced fused image later obtained by using ANN, which eventually increases the act of fusion execution. This work proposes ANN based fusion algorithm for fusing visible and infrared images and obtains better performance by reducing the complexity.
\end{abstract}

Index Terms: Images Fusion, Multi-Scale Decomposition, Artificial Neural Network. Layers.

Keywords: The Rule Aims To Merge These Decomposed

\section{INTRODUCTION}

$D_{\text {igitalized images are a type of data used to address }}$ and simplify problems. The visible light sensors and infrared sensors were commonly recognized by computer vision.

Due to the fast development of image unification mechanics, the infrared sensor notices the radiation of the black body i.e., in the scene, thermal radiation is obtained which lies in a smudged background, that includes an image's poor-quality data. In comparison, visible light sensors may collect greater quantities of spectral data to express precise image information. In less satisfactory scenes, visible sensor images can turn out in a dark and occluded state. The combination of images is now useful for the integration ofvisible and infrared images. The method of combining specifics of more images into one image is a composite of images.

Manuscript received on June 03, 2021.

Revised Manuscript received on June 09, 2021.

Manuscript published on June 30, 2021.

* Correspondence Author

Bhavatharane $\mathbf{K}^{*}$, Student, Department of Computer Science and Engineering, Rashtreeya Vidyalaya College of Engineering, Bengaluru (Karnataka), India. Email: bhavatharanek.scn19@rvce.edu.in

Dr. Minal Moharir, Associate Professor, Department of Computer Science and Engineering, Rashtreeya Vidyalaya College of Engineering, Bengaluru(Karnataka), India. Email: minalmoharir@rvce.edu.in

(C) The Authors. Published by Blue Eyes Intelligence Engineering and Sciences Publication (BEIESP). This is an open access article under the CC BY-NC-ND license (http://creativecommons.org/licenses/by-nc-nd/4.0/)
Combined photographs can provide a scene with more detailed details and are more suitable than any other origin images for successive creation activities. The combined outcome must comply with separate requirements, such as hanging on to all relevant and prominent origin image information. Combined images generate high-quality images providing additional data for simplification, categorization, sectionalization, compression, etc. The fusion mission specification is to decide the most relevant features of the input image and produce fused images without losing detailed information. The key purpose of this work is to merge the infrared (IR) and visible images for surveillance applications to get an improved view. The visual image and image-based fusion of infrared light is an extensive approach to fusion execution in multi-sensor information. Infrared photographs can collect thermal radiation information in areas where it cannot be directly detected by the human eye. In low light, blocking light and damaging weather conditions, they can view objects more accurately. Typically, the visual representations provide greater descriptions of the area within the visible spectrum. It also presents nature's severity and a comparison to human visual opinions. The sufficient bright response in the infrared spectrum is very unpredictable in the visible range because of the variation in thermal emission and spectral susceptibility. It is also hard to interrupt IR imagery. By fusing infrared and visible details, the merged image obtained allows us to reflect the scene more fully and accurately. Under the conditions, the fusion picture would be more informative to the human point of view ofvisible and infrared images.The paper consist of various sections in which section II is of literature survey, section III deals with methodology, section IV provides with all the experimental results and section $\mathrm{V}$ gives the final conclusion of this paper.

\section{METHODOLOGY}

The proposed system for the combination of infrared and visible images using image fusion and ANN.

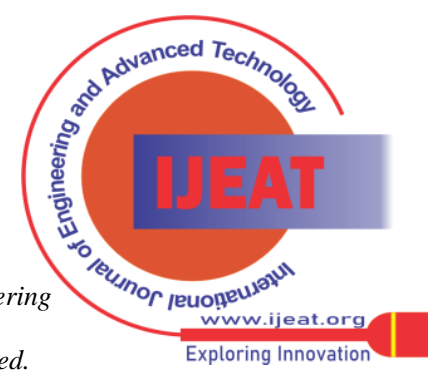




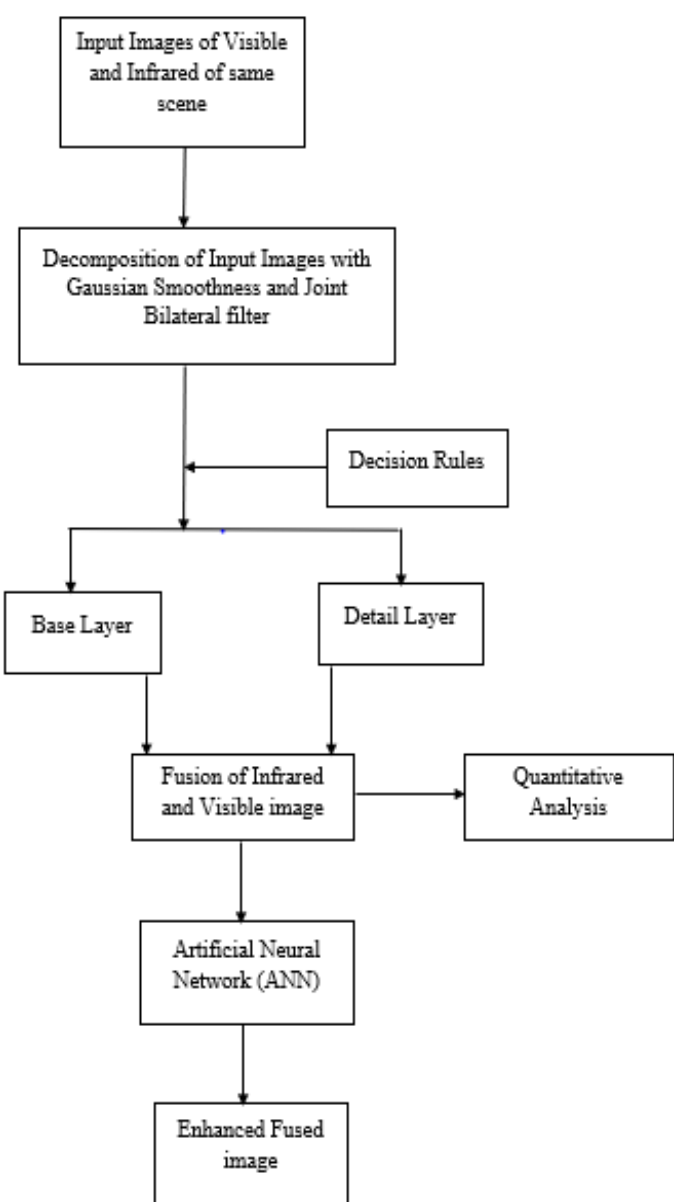

Fig 1: Methodology for Visual and Infrared Image Fusion using ANN

\section{A. MSD-IterationFramework}

Decompose the origin image with Gaussian smoothness and joint bilateral filter. Gaussian filter removes fine scale details whereas joint bilateral filtering helps in extracting edges and the structure details from the source image. As collated with the traditional MSD strategy, decomposition has the characteristics of securing edges and scaling and achieves better fusion performance. Two layers of rules are set to reach the combination of the base layer and the combination of the detailed layers. A saliency map is constructed by "Laplacian and Gaussianfilters" to find the initial weight map for the base layer. Then, guided filters are correlated to decide the final weight map of layer combination. We use theregional average energy weighing to acquire multi-scale decision maps by developing intensity deviation for the detail layer. Through this detailed combination, it can be considered that similarity and sensitivity can satisfy people visual viewpoint. Concluding, the combined base layer and detail layer are reconstructed to get fused image and perform quantitative study to know the fusion quality and performance of the image. The artificial neural network is utilized to create the improved fused picture, which is then compared to the existing approach.

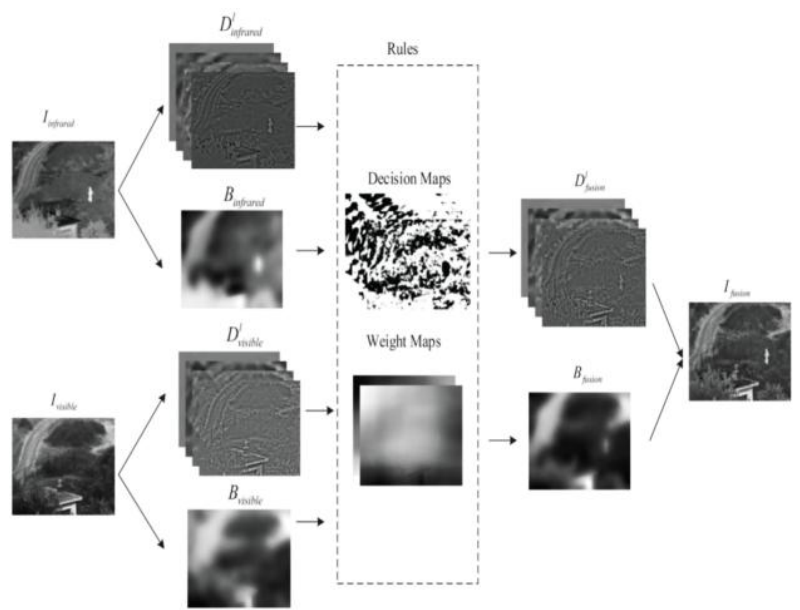

Fig 2: MSD-Iteration Framework. Iinfrared, Ivisible; $D l$ infrared, $D l$ visible, Binfrared, Bvisible: source detail layer, source base layer; $D l$ fusion, Bfusion: fusion detail layers, fusion base layers; Ifusion: fusion image.

\section{B. Moldering the Original Images using Gaussian and Joint Bilateral Filter}

To achieve the good fusion execution, we make use of Gaussian and joint bilateral filters. The filter Gaussian is used for two important aspects namely reduction in noise and smoothing the image. The joint bilateral filter is a nonlinear edge preservation filter that can smooth tiny scale information and detect the image's sides. Edge filters are used to reduce the halos while maintaining spatial stability.This behavior is repetitive process where the restored image is updated iteratively. Multi scale disintegration has the characteristics of edge retention and scale perception to obtain more reliable details from the image. Four level multi scale decomposition iteration is adapted. The benefits of this disintegration are that in order to achieve the proportional perception feature joint bilateral filtering iteration is appealed. The detailed steps are depicted below:

(i) Use the Gaussian filter to remove the fine details of the original images. Apply Gaussian filter iteration to remove the thin formation and smallscale edges to get a smoothened image $\square \square$ by $\square \square=\square \square \square \square \square \square \square \square \quad(\square, \quad \square \square)$ ........................ (1)

Where $\quad \square \square \square \square \square \square \square \square$ represents the Gaussian filter, 'I' is the original image, ' ' denotes standard deviation.

(ii) Iterative extraction of edges and detail structure through joint bilateral filtering. It performs iterations to get the effective images in the modernization and optimization process through detailed structures and edges. Calculate,

$I j=b f i l(I 1, I 1, n, \sigma s, \sigma I)$.

(iii) To get detailed information on multiple scales using Equation (1) and (2) to perform a series of operations, we get the details of multiple scales given in Equations (3) to (5)

$D l=I j l-1-I j l(l=1,2, \ldots N-1)$

$B=\operatorname{Gauss}(I j N-1, \sigma s N)$.

$D N=I j N-1-B$.

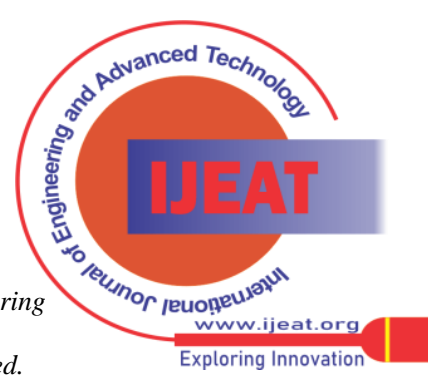


Where $B$ and $D l$ is and base layer and detail layer, " $N$ " is the decomposition number. By iteratively decomposing the source image with Gaussian smoothness and joint bilateral filtering, we obtain the detail layer $D l$ and the base layer B for layer combination. $D l$ represents the detailed feature of the images to transmit highfrequency details on a small structure of images. The basic layer B is an exquisite portion that retains details of rough structure, and used to manage the overall contrast and overall appearance of the composite image. Compared with the traditional multi-scale decomposition technology, the algorithm is decomposed, including edge retention and scale perception features, to protect the separation of structure and details, and improve fusion performance.

\section{Multi Scale Decomposition Iteration}

In the case ofvisible light and infrared images, most functions there is individually in particular proportions. When observing at different scales, the images will have various presentations. As a result, pixel-based image representations are only suited for specific data-level processing, and we frequently need to extract picture characteristics at an adequate scale to verify infrared and visible images.Use two important rules to merge this decomposed layer. The final fused picture is reconstructed using the combined layer. Checkingand identifying the target scene is indeed very beneficial. In order to achieve this goal, it is required to design a two-layer rule combination to improve the fusion quality.

\section{D.Basic Layer Rules}

Binfrared and Bvisibleare assumed to be base layer isolated from infrared and visual images. Implementing a Laplacian filter for each original imageItype (type = infraredorvisible) to achieve a high pass image HPtypeas in Equation (6)

$$
\text { HPtype }=\text { lAP }(\text { Itype }, \bar{m}, \bar{n}) \text {. }
$$

lAP defines the Laplacian filter; $\bar{m}$, $\bar{n}$ is just the size of the filterwhich is usually set to $3 \times 3$. After that, use the local average value of absolute value of HPtypetype as to construct the saliency map SMtype as in Equation (7) SMtype $=(\mid$ HPtype $\mid, k, \sigma g l p)$.

$G L P$ here means Gaussian low pass filter; $\sigma g l p$ is calledstandard deviation. The value of $\mathrm{k}$ and $\sigma$ glpare usually set to 5. SMtype better represents the significance level of detail, which is mainly used to mark the initial weight map IWPtypeas in Equation (8)

IWPtype $(x, y)=(1$, ifSMtype $(x, y)=\max (\operatorname{SMinfrared}(x, y)$, SMvisible $(x, y)) 0$,elsewhere).

Among them (x, y) are only pixels ofIWPtype. However, the initial weight map IWPtype frequently contains noise and is not aligned with the object boundary, which may be passed to the fused picture artefacts. In order to control the above challenges, we use the guided filter IWPtype to obtain the final weight mapFWMtype as in Equation (9)

FWMtype $=$ Gui (IWPtype,Itype, $r, \tau) \ldots \ldots \ldots \ldots \ldots$ (9)

" Guided " defines a guide filter, where " $\mathrm{r}$ " and " $\tau$ " are parameters, and Itype is only the guided image used for the filtering process. As shown in the following formula (10), we obtain the fusion base layer $B f$ usion as in Equation (10) $B$ fusion $=F W$ MinfraredBinfrared $+F W M$ visibleBvisib le .............................................. (10)

Among them, Binfrared andBvisibleportray visual and infrared base layers. For $B$ fusionin (10), to improve the execution of fusion images, the saliency growth and artefact suppression plays a crucial role. 'Saliency' is a visible attribute that reflects people's interest in main areas of the image. The saliency map SMtypein construction (7) is to disclose the level of detail and encourage the saliency development of $B$ fusion. The weight map from (8) the artefacts occur from IWPtype being noisy and not aligned with the object boundary. As a result, we apply spatial consistency to solve this issue. The term "spatial consistency" refers to the tendency for two neighboring pixels with the same intensity to have the same weight. The guided filtering approach is designed to improve the initial weight mapIWPtypeby ensuring spatial consistency.This optimization technique can effectively suppress the appearance of artefacts in the combined image.

\section{E . Detail Layer Rules}

To know about the detail layer rules, use the regional average energy weight to combine these layers. Defining the average energy $\square \square$ deviationl $(x, y)$ and its neighborhood at layer l, as shown in Equation (11).

$$
\text { en_deviation }^{l}(x, y)=\frac{\text { ave_en }_{\text {infrared }}^{l}(x, y)}{\text { ave_en }_{\text {visible }}^{l}(x, y)+\text { ave_en }_{\text {infrared }}^{l}(x, y)}
$$

Select the threshold Th in the range (Th $\in(0.5,1))$ and define the terms $\alpha=\mathrm{Th} / 1+\mathrm{Th}$ and $\beta=(1 / 1+\mathrm{Th})$. The pixels (x,y), if $\alpha<<$ en_deviationl $(x, y) \leq 1 / 2$ or $1 / 2$ $<$ en_deviationl $(x, y)<\beta$, then the average energy is lower deviation. Thus, the infrared image and the visible image have better similarity.Dfusionl $(x, y)$, the detail layers is expressed as in Equation (12).

Dfusionl $(x, y)=(1-a l(x, y))$ Dvisiblel $(x, y)+a l$ $(x, y)$ Dinfraredl $(x, y)$ where $D$ fusionl $(x, y)$ is called detail layer fusion, $a l$ is the decision map of layerl. The final combined image is positioned in the reflection of local energy deviation to examine the similarity between thevisible and infrared images.

\section{F.Artificial Neural Network}

Artificial neural networks have several advantages, but one of the most recognized advantages is the fact that it can be learned from observation data sets [20]. In this approach, the artificial neural network serves as a tool for approximating random functions. These tools aid in determining the most cost-effective and optimum solution while also defining compute functions and enhancing model performance. To achieve a solution, ANN obtains data samples rather than the complete data set, saving time and money.ANNs are basic mathematical models that may be used to improve existing data analysis methods. Three layers of the artificial neural network are interconnected. Input neurons make up the first layer.

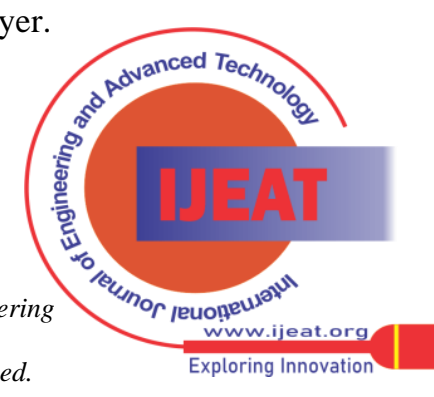


These neurons convey data to the "hidden layer," which in turn sends output neurons to the "output layer," which is the third layer.

\section{G. Performance Analysis}

(i) Entropy

$\mathrm{p}(\mathrm{i}, \mathrm{j})$ is the fused image. en $=\sum(i,) \log (p(i, j)) i, j$ ....(13)

(ii) Edge-dependent Fusion Quality Index $q v a l=\sum|i-j| p(i, j) i, j$

(iii) Mean Square Error (MSE)

$M S E=1 / M N \sum \sum\left((x, y)-f^{\prime}(x, y)\right) 2 N y=1 M x=1 \ldots$ (15)

(iv) Peak Signal-to-Noise ratio (PSNR)

$P S N R=10 \log 10(2 n-1) / \sqrt{ } 1 M N \sum \sum\left((x)-,f^{\prime}(x, y)\right) N 2$ $y=1$

$M x=1$.

(v) Natural Image Quality Evaluator (NIQE)

niqemodel $=$ niqe $(I)$.

(vi) Structural Similarity Index (SSIMVAL)

ssimval $=\operatorname{sim}(C, I)$.

(vii) Information Fidelity Criterion (IFC)

The clear information fidelity criteria are calculated, and the mutual information between the source and the perverted images is measured.

(viii) Blind/Reference less Image Space Quality Evaluator (BRISQUE)

It uses a blind/reference-free image spatial quality evaluator (BRISQUE) to measure the reference-free image quality of combined images.

(ix) BRISQUE Noise

It uses the default image to calculate the BRISQUE noise score of each image and displays the score. The smaller the score, the better the perceived quality.

(x) Mutual Information (MI)

It calculates the mutual information between the two discrete variables of the images.

\section{EXPERIMENTAL RESULTS}

Combining edge retention and scaling perception attributes, it is possible to achieve satisfactory structural isolation based on the details of the image. We must follow the two-layer rules to combine the base layer and the detail layer. The basic layer rules aim to have significant development and artifact suppression on the mixed results, and then build a detail layer rule to satisfy human visual viewpoint. The Objective comparison of the existing method [7] without using ANN i.e., using Gaussian Filtering and joint bilateral filtering iteration.
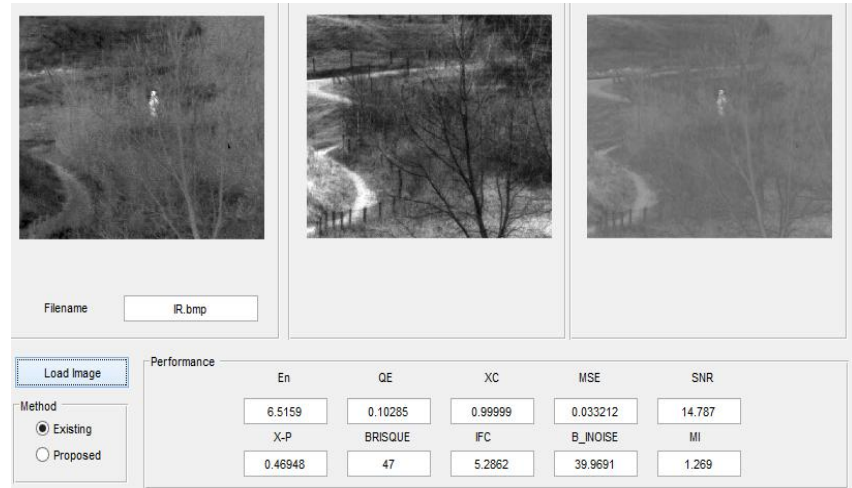

Fig 3: a. UN Camp- Existing method
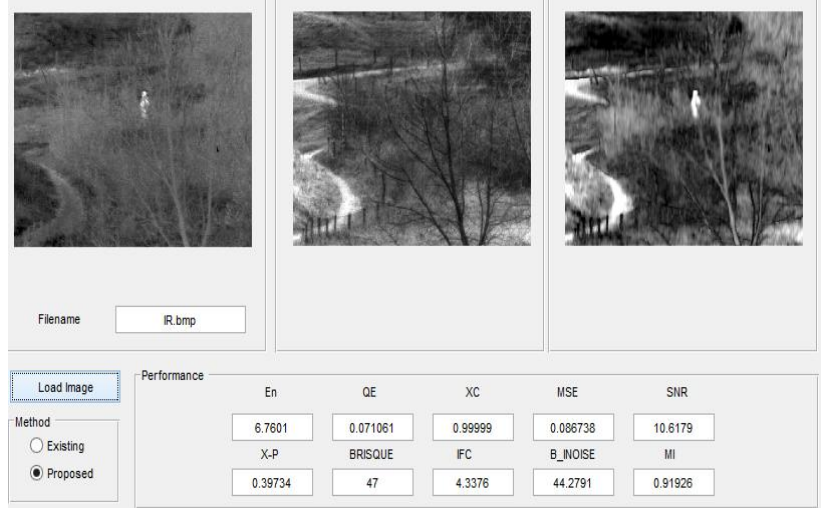

Fig 3: b. UN Camp- Proposed method

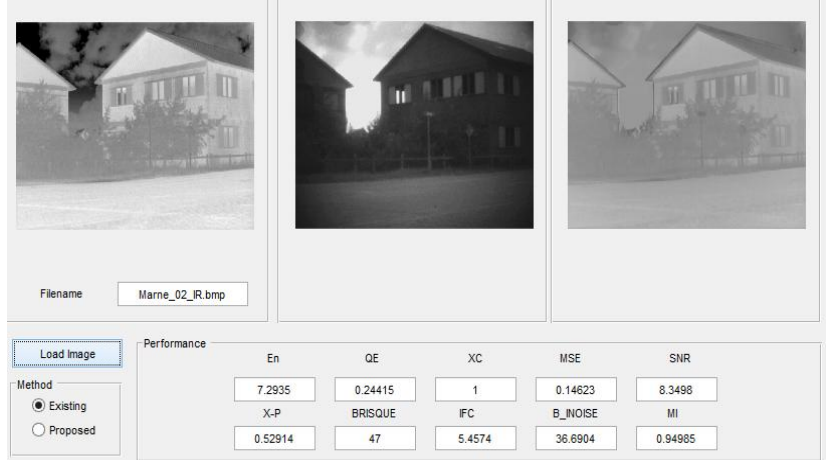

Fig 4: a. Marne-02- Existing method

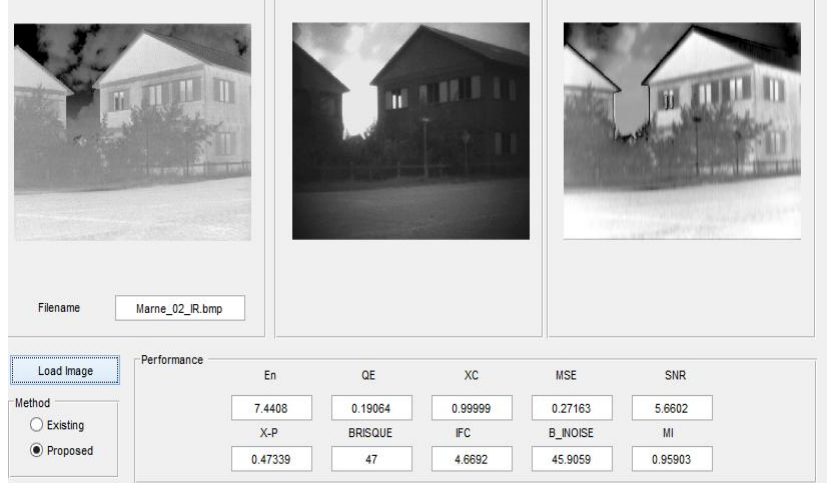

Fig 4: a. Marne-02- Proposed method

Training the Neural Network with the base and detail features obtained from the rule of base layers and detail layers. The function fitting Neural Network is shown in Fig 5. By using the training neural network shown in Fig 6, fused image is obtained.

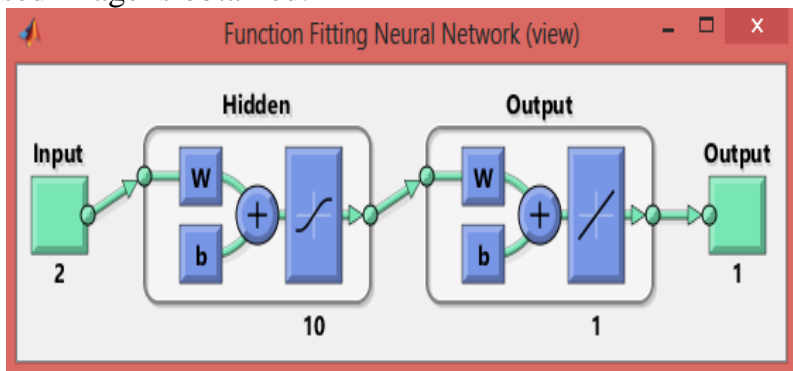

Fig 5: Function Fitting Neural Network

Published By:

Blue Eyes Intelligence Engineering and Sciences Publication

(C) Copyright: All rights reserved.

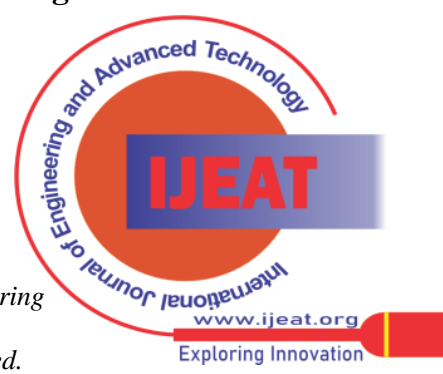




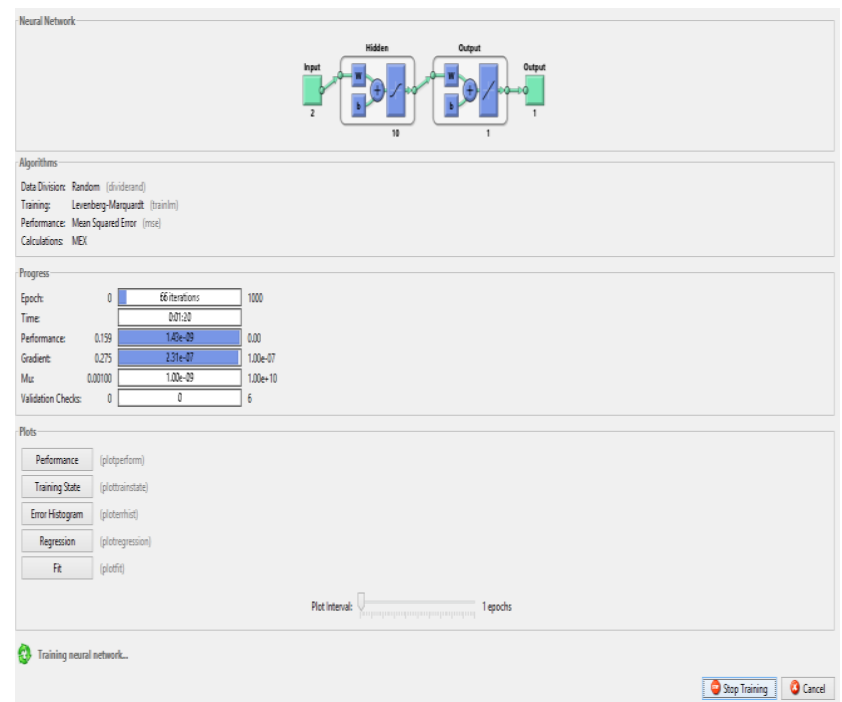

Fig 6: Training the Neural Network

\begin{tabular}{|l|c|c|c|c|c|c|c|c|}
\hline & \multicolumn{5}{|c|}{ Existing Method } & \multicolumn{4}{|c|}{ Proposed Method } \\
\hline Parameters & $\begin{array}{c}\text { UN } \\
\text { Camp }\end{array}$ & Marne & Kaptein & Tank & $\begin{array}{c}\text { UN } \\
\text { Camp }\end{array}$ & Marne & Kaptein & Tank \\
\hline En & 6.3159 & 7.0935 & 6.852 & 7.1294 & 6.7601 & 7.4408 & 7.3508 & 7.6326 \\
\hline QE & 0.1025 & 0.2441 & 0.1586 & 0.10925 & 0.0710 & 0.1906 & 0.1356 & 0.0632 \\
\hline Xc & 0.9999 & 1 & 0.9999 & 0.9999 & 0.9999 & 0.9999 & 0.9999 & 0.9999 \\
\hline MSE & 0.0332 & 0.1462 & 0.05288 & 0.0638 & 0.0867 & 0.27163 & 0.1046 & 0.0641 \\
\hline SNR & 14.787 & 8.3498 & 12.781 & 11.9796 & 10.6179 & 5.6602 & 9.8042 & 11.9298 \\
\hline x-p & 0.4694 & 0.5291 & 0.5201 & 0.4052 & 0.8973 & 0.4733 & 0.5548 & 0.3460 \\
\hline BRISQUE & 47 & 47 & 47 & 47 & 47 & 47 & 47 & 47 \\
\hline IFC & 5.2862 & 5.4574 & 4.7429 & 5.706 & 4.3376 & 4.6692 & 4.5784 & 6.4057 \\
\hline B_NOISE & 39.969 & 36.6904 & 38.125 & 37.735 & 44.2791 & 45.9059 & 40.614 & 43.9654 \\
\hline MI & 1.269 & 0.9485 & 1.034 & 1.1659 & 0.9192 & 0.95903 & 1.713 & 1.9164 \\
\hline
\end{tabular}

Table 1: Objective Results of Proposed Method and Existing Method

The performance of the method is calculated by the objective metrics such as "entropy En, Xydeas-Petrovic X$\mathrm{P}$, edge dependent fusion quality index $\mathrm{QE}$, information fidelity criterion IFC, mean square error MSE, peak signalto-noise ratio SNR, naturalness image quality evaluator NIQE, Structural similarity index for image quality SSIM and brisque parameter". Which is represented in Table 1 for visual images and infrared contain UN Camp, Marne-02, Kaptein-01, Octec, Tank and Road are some of the examples acquired the data from the datasets [15].

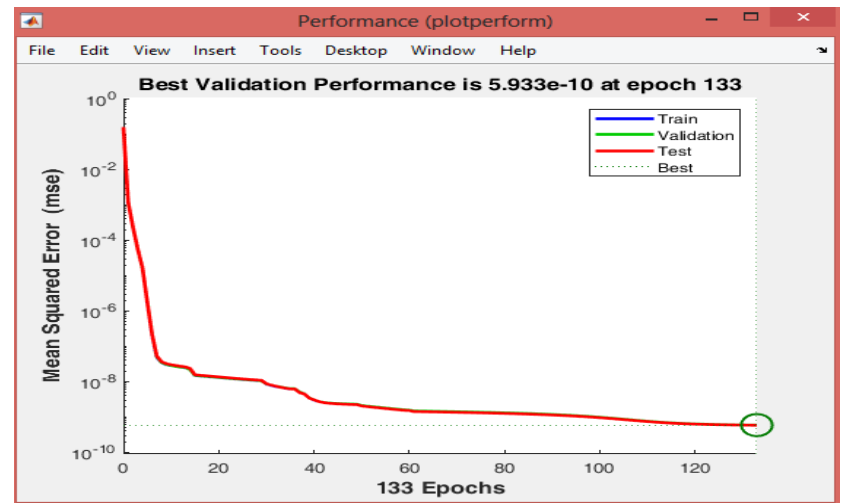

Fig 7: Performance Plot

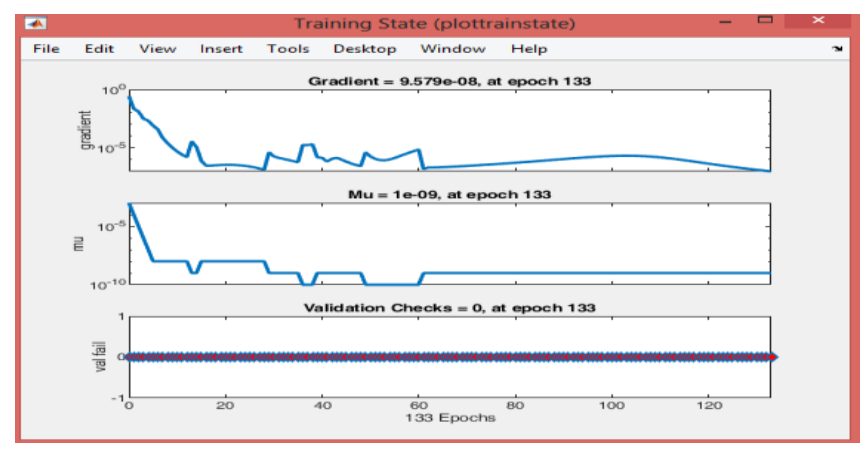

Fig 8: Plot of Training State

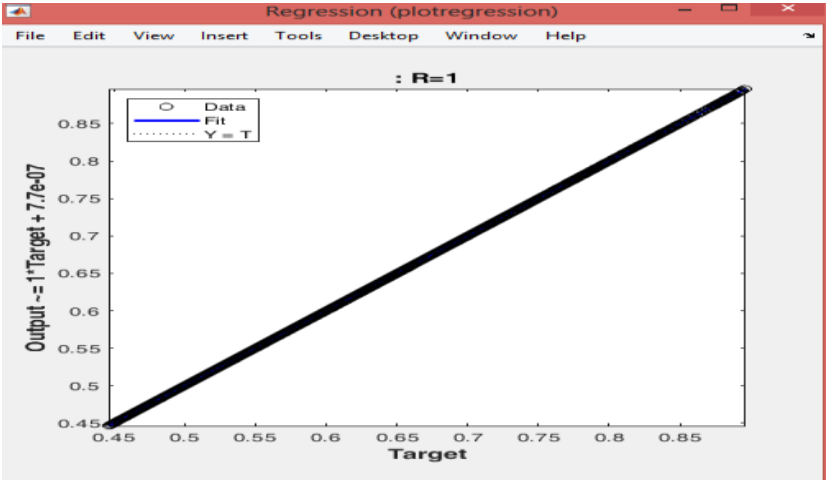

Fig 9: Regression Plot

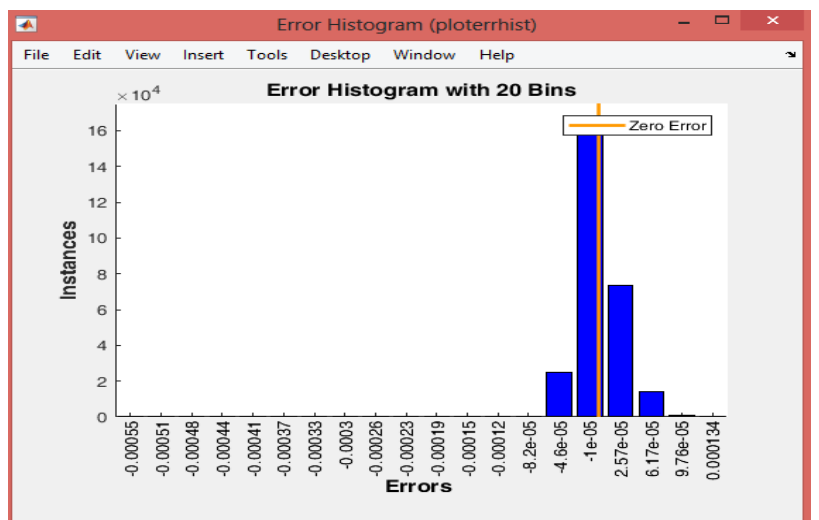

Fig 10: Error Histogram Plot

The performance graph of the method using ANN is shown in Figure 7. This diagram allows users to understand the current status of the training process. The $\mathrm{X}$-axis represents the number of iterations (133 epochs). In Figure 8 the $\mathrm{Y}$ axis represents the MSE that occurs at each iteration. The regression graph and error histogram graph are shown in Figures 9 and Figure 10.

\section{CONCLUSION}

This work proposed a framework for iterative methods using multi-scale decomposition of visual light and infrared image fusion based on artificial neural network. The benefits of this MSD iterative method are that it can be decomposed by "Gaussian smoothness and joint bilateral filters". The method is described in detail using a Gaussian filter for finescale detail extraction and joint bilateral filtering for edge and structure extraction.

Published By:

Blue Eyes Intelligence Engineering and Sciences Publication

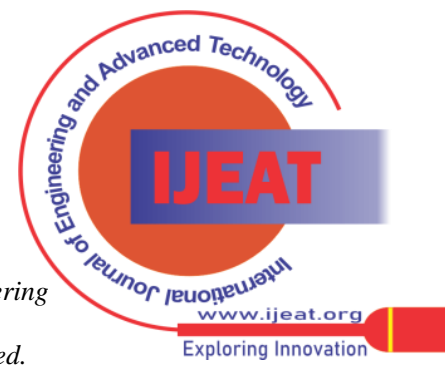


Decomposition includes preserving the attributes of edge and zoom perception, so that the detail information is completely separated from the image details and the improvement of fusion performance is maintained. The rule aims to merge these decomposed layers. A saliency map is constructed by Laplacian and Gaussian filters to find the initial weight map, and the guided filter is further used to determine the final weight map. Basic layer rules can actually increase saliency and conquer the appearance of artefacts. Especially for the rules of the detail layer, we actually use it in combination with the regional average energy weighting to acquire the decision graph by forming intensity deviations and combining the images. Thus, ensuring the beneficial details about achievements and clear human visual perception. The enhanced fused image later obtained by using ANN, which eventually increases the act of fusion execution. This work proposes ANN based fusion algorithm for fusing visible and infrared images and obtains better performance by reducing the complexity. The proposed framework has been widely measured on "UN Camp, Road, Marne-02, Octec, Kaptein-1654, and Marne”.

\section{REFERENCES}

1. Liu, Yu, Shuping Liu, and Zengfu Wang. "A general framework for image fusion based on multi-scale transform and sparse representation." Information Fusion 24 (2015): 147-164.

2. Shi, Zaifeng, Jiangtao Xu, Jufeng Zhao, and Qing Xin. "Fusion for visible and infrared images using visual weight analysis and bilateral filter-based multi scale decomposition." Infrared Physics \& Technology 71 (2015): 363-369.

3. Zhao, Jufeng, Qiang Zhou, Yueting Chen, Huajun Feng, Zhihai Xu, and Qi Li. "Fusion of visible and infrared images using saliency analysis and detail preserving based image decomposition." Infrared physics \& technology 56 (2013): 93-99.

4. Lewis, John J., Robert J. O’Callaghan, Stavri G. Nikolov, David R. Bull, and Nishan Canagarajah. "Pixel level and region-based image fusion with complexwavelets." Information fusion 8, no. 2 (2007): 119-130.

5. Ma, Jinlei, Zhiqiang Zhou, Bo Wang, and Hua Zong. "Infrared and visible image fusion based on visual saliency map and weighted least square optimization." Infrared Physics \& Technology 82 (2017): 8-17.

6. Li, Shutao, Bin Yang, and Jianwen Hu. "Performance comparison of different multiresolution transforms for image fusion." Information Fusion 12, no. 2 (2011): 74-84.

7. Xing, Changda, Zhisheng Wang, Fanliang Meng, and Chong Dong. "Fusion of infrared and visible images with Gaussian smoothness and joint bilateral filtering iteration decomposition." IET Computer Vision 13, no. 1 (2018): 44-52.

8. Pan, Lili, Mei Xie, and Zheng Ma. "Iris localization based on multiresolution analysis." In 2008 19th International Conference on Pattern Recognition, pp. 1-4. IEEE, 2008.

9. Zhou, Zhiqiang, Bo Wang, Sun Li, and Mingjie Dong. "Perceptual fusion of infrared and visible images through a hybrid multi-scale decomposition with Gaussian and bilateral filters." Information Fusion 30 (2016): 15-26.

10. Zhang, Yu, Lijia Zhang, Xiangzhi Bai, and Li Zhang. "Infrared and visual image fusion through infrared feature extraction and visual information preservation." Infrared Physics \& Technology 83 (2017): 227-237.

11. Zhu, Pan, Xiaoqing Ma, and Zhanhua Huang. "Fusion of infraredvisible images using improved multi-scale top-hat transform and suitable fusion rules." Infrared Physics \& Technology 81 (2017): 282 295.

12. Bavirisetti, Durga Prasad, and Ravindra Dhuli. "Two-scale image fusion of visible and infrared images using saliency detection." Infrared Physics \& Technology 76 (2016): 52-64.

13. Zhao, Jufeng, Yueting Chen, Huajun Feng, Zhihai Xu, and Qi Li. "Infrared image enhancement through saliency feature analysis based on multi-scale decomposition." Infrared Physics \& Technology 62 (2014): 86-93.

Bhavatharane $\mathbf{K}$, is aMTech student at Department of Computer Science and Engineering, Rashtreeya Vidyalaya college of Engineering, Bengaluru, Karnataka. bhavatharanek.scn19@rvce.edu.in

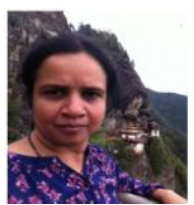

Dr. Minal Moharir, is working as Associate professor Department of Computer Science and Engineering, Rashtreeya Vidyalaya College of Engineering, Bengaluru, Karnataka, India.minalmoharir@rvce.edu.in

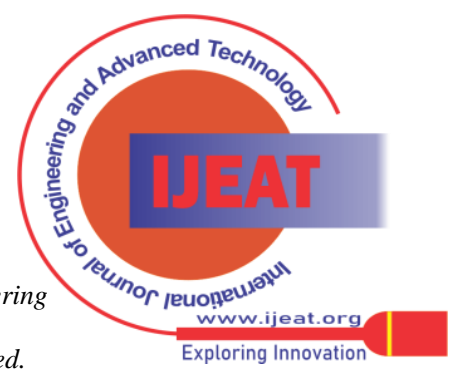

